\title{
A new pressure relaxation closure model for one-dimensional two-material Lagrangian hydrodynamics
}

\author{
J.R. Kamm ${ }^{1, a}$, M.J. Shashkov ${ }^{2}$, and W.J. Rider ${ }^{1}$ \\ 1 Sandia National Laboratories, Albuquerque, NM 87185, USA \\ 2 Los Alamos National Laboratory, Los Alamos, NM 87545, USA
}

\begin{abstract}
We present a new model for closing a system of Lagrangian hydrodynamics equations for a two-material cell with a single velocity model. We describe a new approach that is motivated by earlier work of Delov and Sadchikov and of Goncharov and Yanilkin. Using a linearized Riemann problem to initialize volume fraction changes, we require that each material satisfy its own $p d V$ equation, which breaks the overall energy balance in the mixed cell. To enforce this balance, we redistribute the energy discrepancy by assuming that the corresponding pressure change in each material is equal. This multiple-material model is packaged as part of a two-step time integration scheme. We compare results of our approach with other models and with corresponding pure-material calculations, on two-material test problems with ideal-gas or stiffened-gas equations of state.
\end{abstract}

\section{Introduction}

We present a new model for closing a system of Lagrangian hydrodynamics equations for a twomaterial cell with a single velocity model. In Arbitrary Lagrangian-Eulerian (ALE) methods [5] with several materials, multiple-material Lagrangian cells invariably arise when the flow field is remapped onto a new mesh. There are several existing models for this problem. The method of Tipton (see [9]) is an explicit relaxation model that adds a viscosity-like term to the pressure of each material. Equality of all materials' augmented pressures and preservation of volume imply solutions for the volume changes, which are used to update the states of the mixed-cell materials. The method of Kamm and Shashkov [6] is based on the instantaneous pressure-equlibration model of Després and Lagoutière [3]. Instead of instantaneous equilibration, Kamm and Shashkov (K\&S) use a relaxation procedure based on an optimization problem that uses the solution of a local Riemann problem. Delov and Sadchikov [2] and Goncharov and Yanilkin [4], estimate the velocity of the sub-cell interface between materials, which suggests an expression for the volume change of each material. The internal energy for each material is updated from its own $p d V$ equation; this update contains an exchange term between the mixed cell materials. Motivated by this approach, Kamm et al. [7] propose an internal energy update that perforce satisfies the overall mixed-cell energy balance equation. Here, we describe a new approach that evolved from these earlier methods, in which we adjust the materials' specific internal energies, to drive the mixed cell toward pressure equilibrium. Section 2 contains a review of the basic 1D Lagrangian hydrodynamics equations and a description of the predictor-corrector schemes used to obtain solutions. In $\S 3$, we describe our new pressure-relaxation model. Test problems and results for this method are provided in $\S 4$. We summarize our findings and conclude in $§ 5$.

\footnotetext{
a e-mail: jrkamm@sandia.gov
}

This is an Open Access article distributed under the terms of the Creative Commons Attribution-Noncommercial License 3.0, which permits unrestricted use, distribution, and reproduction in any noncommercial medium, provided the original work is properly cited. 


\section{Lagrangian hydrodynamics in 1D}

The partial differential equations governing the balance of momentum and internal energy, written in the Lagrangian frame of reference, are:

$$
\begin{gathered}
\rho \frac{d u}{d t}+\nabla P=0, \\
\rho \frac{d \varepsilon}{d t}+P \nabla \cdot u=0 .
\end{gathered}
$$

Here, $u$ is the velocity and $P=P(\tau, \varepsilon)$ is the thermodynamic pressure, where $\varepsilon$ is the specific internal energy (SIE) and $\tau$ is the specific volume, equal to the inverse of the mass density $\rho$. The mass of a fluid parcel is constant, so that $\tau$ is the volume of that parcel divided by its mass. In this section, subscripts denote spatial position and superscripts indicate temporal indexing. In our staggered-mesh discretization, cell-centers (at index $i+1 / 2$ ) are associated with cell masses $M_{i+1 / 2}$, cell volumes $V_{i+1 / 2}$, and thermodynamic state variables, such as density $\rho_{i+1 / 2}$, specific volume $\tau_{i+1 / 2}$, SIE $\varepsilon_{i+1 / 2}$, pressure $p_{i+1 / 2}$, and sound speed $c_{s, i+1 / 2}$. The vertices of cell $i$ are associated with edge positions $x_{i}$ and $x_{i+1}$, edge velocities $u_{i}$ and $u_{i+1}$, and node-centered control volume masses $m_{i}$ and $m_{i+1}$. The volumes are determined from the edge positions, which evolve according to the trajectory equation,

$$
d x_{i} / d t=u_{i}
$$

We assume that we have the necessary information to specify all state variables at time $t^{n}$ and seek to update the solution to time $t^{n+1} \equiv t^{n}+\delta t$. The following algorithm for integrating the pure-material equations is used in our software implementation. This scheme is stable under the usual constraints, e.g., on the CFL number, and is nominally second-order accurate in both space and time for smooth initial conditions and sufficiently short times, degenerating to first order as discontinuities develop. In the first phase, the assignment of the pressure is based on an adiabatic relation. The full EOS call in the second phase is retained, to ensure thermodynamic consistency, conservation, and accuracy at the updated time. Certain quantities are first updated to the half-timestep level $(H)$, and then all quantities are updated to the full timestep $(F)$.

$$
\begin{array}{cc}
\text { Half-timestep } & \begin{array}{c}
\text { Full-timestep } \\
u_{i}^{n+1}=u_{i}^{n}-\frac{\delta t}{m_{i}}\left(p_{i+1 / 2}^{n+1 / 2}-p_{i-1 / 2}^{n+1 / 2}\right)
\end{array} \\
x_{i}^{n+1 / 2}=x_{i}^{n}+(\delta t / 2) u_{i}^{n} & x_{i}^{n+1}=x_{i}^{n}+\frac{\delta t}{2}\left(u_{i}^{n}+u_{i}^{n+1}\right) \\
V_{i+1 / 2}^{n+1 / 2}=x_{i+1}^{n+1 / 2}-x_{i}^{n+1 / 2} & V_{i+1 / 2}^{n+1}=x_{i+1}^{n+1}-x_{i}^{n+1} \\
\tau_{i+1 / 2}^{n+1 / 2}=V_{i+1 / 2}^{n+1 / 2} / M_{i+1 / 2} & \tau_{i+1 / 2}^{n+1}=V_{i+1 / 2}^{n+1} / M_{i+1 / 2} \\
p_{i+1 / 2}^{n+1 / 2}=p_{i+1 / 2}^{n} \\
-\left(\left(c_{s, i+1 / 2}^{n}\right)^{2} / \tau_{i+1 / 2}^{n}\right)\left(\delta V_{i+1 / 2}^{n+1 / 2} / V_{i+1 / 2}^{n}\right)
\end{array}
$$

In $(8 F)$ and $(9 H)$, the change in volume is $\delta V_{i+1 / 2}^{n+j} \equiv V_{i+1 / 2}^{n+j}-V_{i+1 / 2}^{n}$ with $j=1$ or $1 / 2$, respectively. The pressure in these expressions (e.g., in $(4 F)$ and $(8 F)$ ) is augmented by an artificial viscosity $q_{i}^{n}$ to provide additional numerical dissipation, e.g., at shock waves. We use the the classical linearplus-quadratic model, active only in compression, for $q^{n}$; this simple model is sufficient for demonstration purposes. 


\section{Two-material pressure relaxation model}

Our new pressure relaxation model builds upon the sub-scale dynamics (SSD) approach described by Kamm et al. [7], which was inspired by the work of Delov and Sadchikov [2] and Goncharov and Yanilkin [4]. In 1D, the velocity of the sub-cell material interface, $u_{\text {intfc }},{ }^{1}$ is given by the linearized Riemann-solver approximation as:

$$
u_{\mathrm{intfc}}=\frac{\rho_{1} c_{s, 1} u_{1}^{-}+\rho_{2} c_{s, 2} u_{2}^{+}+\left(p_{1}-p_{2}\right)}{\rho_{1} c_{s, 1}+\rho_{2} c_{s, 2}},
$$

where $u_{1}^{-}, u_{2}^{+}$are at the immediate left and right of the interface, respectively. Assuming that the velocity varies linearly within the cell, it follows that

$$
u_{1}^{-}=u_{2}^{+}=f_{2} u_{1}+f_{1} u_{2}
$$

where the unsuperscripted velocities on the RHS of (11) are the values at the cell edges, i.e., $u_{1}=u_{i_{\text {mix }}}$ and $u_{2}=u_{i_{\text {mix }}+1}$. Substituting into (10) and simplifying, one obtains

$$
u_{\text {intfc }}=f_{2} u_{1}+f_{1} u_{2}+\frac{p_{1}-p_{2}}{\rho_{1} c_{s, 1}+\rho_{2} c_{s, 2}},
$$

with the corresponding volume changes of the materials

$$
\delta V_{1}=\left(u_{\text {intfc }}-u_{1}\right) \delta t \quad \text { and } \quad \delta V_{2}=\left(u_{2}-u_{\text {intfc }}\right) \delta t .
$$

Substituting (12) into (13) implies the volume changes:

$$
\delta V_{1}=\beta_{1} \delta V+\frac{\Delta p_{1,2}}{2 \bar{\kappa}} \delta t, \quad \delta V_{2}=\beta_{2} \delta V-\frac{\Delta p_{1,2}}{2 \bar{\kappa}} \delta t
$$

where $\Delta p_{1,2} \equiv p_{1}-p_{2}, \bar{\kappa} \equiv\left(\kappa_{1}+\kappa_{2}\right) / 2, \kappa_{k} \equiv \rho_{k} c_{s, k}$, the $\beta_{k}$ satisfy $\beta_{k} \geq 0$ with $\sum_{k} \beta_{k}=1$, and the volume change of the entire cell over the timestep is $\delta V \equiv\left(u_{2}-u_{1}\right) \delta t$.

Motivated by these expressions, in the predictor phase we follow $(5 H, 6 H)$ for overall mixed-cell values and assign the individual predictor volume updates as

$$
\delta V_{\left\{\begin{array}{l}
1 \\
2
\end{array}\right\}}^{n+1 / 2}=\beta_{\left\{\begin{array}{l}
1 \\
2
\end{array}\right\}}^{n} \delta V^{n+1 / 2} \pm \frac{\Delta p_{1,2}^{n}}{2 \bar{\kappa}^{n}} \frac{\delta t}{2} .
$$

We update the individual materials' volumes, $V_{k}^{n+1 / 2}=V_{k}^{n}+\delta V_{k}^{n+1 / 2}$, volume fractions, $f_{k}^{n+1 / 2}=$ $V_{k}^{n+1 / 2} / V^{n+1 / 2}$, corresponding values of $\beta_{k}^{n+1 / 2}$, and pressures,

$$
p_{k}^{n+1 / 2}=p_{k}^{n}-\left(\left(c_{s, k}^{n}\right)^{2} / \tau_{k}^{n}\right)\left(\delta V_{k}^{n+1 / 2} / V_{k}^{n}\right)
$$

The overall cell pressure is the $\beta$-weighted sum of the individual pressures, $p_{i_{\text {mix }}+1 / 2}^{n+1 / 2}=\sum_{k} \beta_{k}^{n+1 / 2} p_{k}^{n+1 / 2}$.

In the corrector step, the velocities $u_{i_{\text {mix }}}^{n+1}$ and $u_{i_{\text {mix }}+1}^{n+1}$ are computed from $(4 F)$ using the nearby pressures. The values at $t^{n+1}$ of the overall mixed-cell positions and volume are calculated as in $(5 F, 6 F)$. The individual volume changes of the materials are assigned similar to (15), but using (i) volume fractions and pressures from (16) and (ii) the full timestep, $\delta t$ (not $\delta t / 2$ ). We update to the final time the individual materials' volumes, $V_{k}^{n+1}=V_{k}^{n}+\delta V_{k}^{n+1}$, volume fractions, $f_{k}^{n+1}=V_{k}^{n+1} / V^{n+1}$, corresponding values of $\beta_{k}^{n+1}$, and specific volumes, $\tau_{k}^{n+1}=V_{k}^{n+1} / m_{k}$. The corrector SIE update suggested by $(8 F)$ leads to the following provisional SIE values:

$$
\varepsilon_{\left\{\begin{array}{l}
1 \\
2
\end{array}\right\}}^{n+1, \star}=\varepsilon_{\left\{\begin{array}{l}
1 \\
2
\end{array}\right\}}^{n}-\beta_{\left\{\begin{array}{l}
1 \\
2
\end{array}\right\}}^{n+1 / 2}\left(p_{\left\{\begin{array}{l}
1 \\
2
\end{array}\right\}}^{n+1 / 2}+q^{n}\right) d V_{\left\{\begin{array}{l}
1 \\
2
\end{array}\right\}}^{n+1} / m_{\left\{\begin{array}{l}
1 \\
2
\end{array}\right\}} \mp p_{\left\{\begin{array}{l}
1 \\
2
\end{array}\right\}}^{n+1 / 2} \frac{\Delta p_{1,2}^{n+1 / 2}}{2, \bar{\kappa}_{1,2}^{n+1 / 2}} \frac{\delta t}{m_{\left\{\begin{array}{l}
1 \\
2
\end{array}\right\}}} .
$$

\footnotetext{
${ }^{1}$ The quantity $u_{\text {intic }}$ is the velocity of the interface, not the flow velocity $a t$ the interface.
} 


\section{EPJ Web of Conferences}

We call these values "provisional" because this updated internal energy is not conservative, i.e., the overall energy balance equation in the mixed cell is not satisfied:

$$
\sum_{k} m_{k} d \varepsilon_{k}^{n+1, \star} \neq-p^{n+1 / 2} \delta V^{n+1}
$$

To enforce this critical conservation requirement, we first identify the total (not specific) internal energy discrepancy, $\delta \mathcal{E}^{n+1 / 2}$, which, after some algebra, can be written as:

$$
\delta \mathcal{E}^{n+1 / 2}=\sum_{k} m_{k} d \tilde{\varepsilon}_{k}^{n+1 / 2}=\frac{\left(p_{1}^{n+1 / 2}-p_{2}^{n+1 / 2}\right)^{2}}{\kappa_{1}^{n+1 / 2}+\kappa_{2}^{n+1 / 2}} \delta t,
$$

where $d \widetilde{\varepsilon}_{k}^{n+1 / 2}$ is the additional SIE increment in material $k$ that ensures conservation. To distribute this energy among the materials, we make two further assumptions: (i) the pressure change that corresponds to this energy discrepancy, $d \tilde{P}$, is the same in both materials; and (ii) this pressure change occurs with no further change in density. These assumptions imply the following equations for $d \tilde{P}$ :

$$
d \tilde{P}=\left(\partial p_{1} / \partial \varepsilon_{1}\right)_{\rho_{1}} d \tilde{\varepsilon}_{1} \quad \text { and } \quad d \tilde{P}=\left(\partial p_{2} / \partial \varepsilon_{2}\right)_{\rho_{2}} d \tilde{\varepsilon}_{2}
$$

In summary, we have three unknowns: $d \tilde{\varepsilon}_{1}, d \tilde{\varepsilon}_{2}$, and $d \tilde{P}$ that satisfy the three equations in $(19,20)$. We evaluate the pressure derivatives in (20) with EOS calls, using the updated densities $\rho_{k}^{n+1}$ and provisionally updated SIEs $\varepsilon_{k}^{n+1, \star}$. This allows us to numerically evaluate $d \tilde{P}^{n+1}$ as:

$$
d \tilde{P}^{n+1}=\frac{\delta \mathcal{E}^{n+1 / 2}}{\left[m_{1} /\left(\partial p_{1} / \partial \varepsilon_{1}\right)_{\rho_{1}}^{n+1}\right]+\left[m_{2} /\left(\partial p_{2} / \partial \varepsilon_{2}\right)_{\rho_{2}}^{n+1}\right]}
$$

From (20), we then evaluate the individual SIE increments and use those values to correct the individual SIEs:

$$
\varepsilon_{k}^{n+1}=\varepsilon_{k}^{n+1, \star}+d \widetilde{\varepsilon}_{k}^{n+1} .
$$

We update the pressure in each material with EOS calls,

$$
p_{k}^{n+1}=\mathcal{P}_{k}\left(\tau_{k}^{n+1}, \varepsilon_{k}^{n+1}\right),
$$

leading to self-consistent states for both materials in the mixed cell. There is significant flexibility in specifying details of this method; the algorithm described above covers just one possible approach. We now turn to results.

\section{Test problems and results}

We consider two different test problems with exact solutions, with which to evaluate the method described above. In the mixed-cell test problems, the mesh has $N_{x}$ zones, each of initial dimension $1 /\left(N_{x}+1\right)$, except for a single multi-material zone of initial width $2 /\left(N_{x}+1\right)$. In that zone, the mass and volume fractions are assigned to be consistent with the initial conditions, with the initial material interface located at the geometric center of the cell. We compare results of the new method (identified as KSR after the authors) on these problems with the models of Tipton [9], Kamm and Shashkov $(\mathrm{K} \& \mathrm{~S})$ [6], and Kamm et al.'s sub-scale dynamics (SSD) [7]. We also perform a pure-material calculation (with no mixed cell), with all cells initially the same width; ${ }^{2}$ these calculations contain one more cell than the multi-material calculations: the multi-material zone is divided into two equal-sized

\footnotetext{
${ }^{2}$ We set up the test problems this way in order to compare, as closely as possible, to the pure-material calculations.
} 
pure-material zones for a total of $N_{x}+1$ zones, each of width $1 /\left(N_{x}+1\right)$. All problems were run with the same value of CFL constant, equal to 0.5 . We provide results for the time-histories of pressure in the multi-material cell or, in the pure-material calculation, adjacent to the interface.

The first test problem we consider is the modified Sod shock tube problem variant of Barlow [1] (see also [9]), with a final time of $t_{\text {final }}=0.2$ and the following array of non-dimensional initial conditions for $\{\gamma, \rho, \varepsilon, p, u\}$ :

$$
\begin{array}{cc}
\{2,1,2,2,0\}, & \text { if } 0<x<0.5, \\
\{1.4,0.125,2,0.1,0\}, & \text { if } 0.5<x<1,
\end{array}
$$

where $p=(\gamma-1) \rho \varepsilon$. The mixed cell initially contains both of these distinct states.

The second test problem is the water-air shock tube, a standard problem for multi-material compressible flow [8]. It tests inherently compressible flow features, uses a slightly more complicated and stiffer EOS, and has a directly computable solution. Here, water is described by the stiffened-gas EOS with $p=(\gamma-1) \rho \varepsilon-\gamma p_{\infty}$. The initial conditions for the array $\left\{\gamma, p_{\infty}, \rho, \varepsilon, p, u\right\}$, in mks units, are:

$$
\begin{array}{ll}
\left\{4.4,6 \times 10^{8}, 10^{3}, 1.07 \times 10^{6}, 10^{9}, 0\right\}, & \text { if } 0<x<0.7, \\
\left.1.4,0, \quad 50,5 \times 10^{4}, \quad 10^{6}, 0\right\}, & \text { if } 0.7<x<1,
\end{array}
$$

with a final time of $t_{\text {final }}=2.2 \times 10^{-4} \mathrm{~s}$. The multi-material cell is initially centered at $x=0.7$.

Plots of the pressure time-histories in the mixed cell are shown in Fig. 1 for the modified Sod problem with $N_{x}=99$ (left column) and the water-air shock tube problem with $N_{x}=249$ (right column). In both problems, the nature of the pressure-equilibration varies among the different methods; all models ultimately arrive at the exact solution result at the final time. For modified Sod, the KSR time-histories results are very close to the SSD results. Both methods exhibit behavior suggestive of an optimally-damped configuration, as opposed to the other models' results, which equilibrate more slowly. For the water-air shock tube, the KSR result equilibrates without achieving any negative pressure state in material 1 (the solid line), which is true for the K\&S result but not true for the other models. The K\&S approach is complicated and computationally more intensive, while the current approach is clearer, simpler, and faster.

\section{Summary and conclusions}

We have presented a new model for closing the system of equations for a two-material cell under the single velocity, single pressure assumption in one dimensional Lagrangian hydrodynamics. The materials in the mixed cells are treated as distinct, presenting the problem of how to assign the thermodynamic states of the individual materials together with the nodal forces that this zone generates. The new model, dubbed KSR, evolved from the SSD model of Kamm et al. [7] and was motivated by the work of Delov and Sadchikov [2] and of Goncharov and Yanilkin [4]. The key concept is to use the individual materials' energy balance equations, to which an additional energy term is added, by which the energy balance of the mixed materials is guaranteed to equal that of the overall cell values. The preliminary KSR results on these problems are promising: they exhibit similar favorable characteristics (e.g., near-optimal pressure damping and pressure positivity) of the more complicated $K \& S$ method, but with algorithmic simplicity comparable to that of the SSD approach. There are additional 1D test problems on which these methods should be evaluated, including higher Mach numbers and very small initial volume fractions. We are devising methods based on this approach for multi-material cells in the 2D and 3D Lagrangian hydrodynamics equations. Furthermore, this approach should be generalized for material strength effects.

This work, available as Sandia Report 2010-2962J, was performed under the auspices of the National Nuclear Security Administration of the United States Department of Energy by Sandia National Laboratories, a multiprogram laboratory operated by Sandia Corporation, a Lockheed-Martin Company, under contract DE-AC94AL85000, and by Los Alamos National Security, LLC, at Los Alamos National Laboratory under contract 


\section{EPJ Web of Conferences}
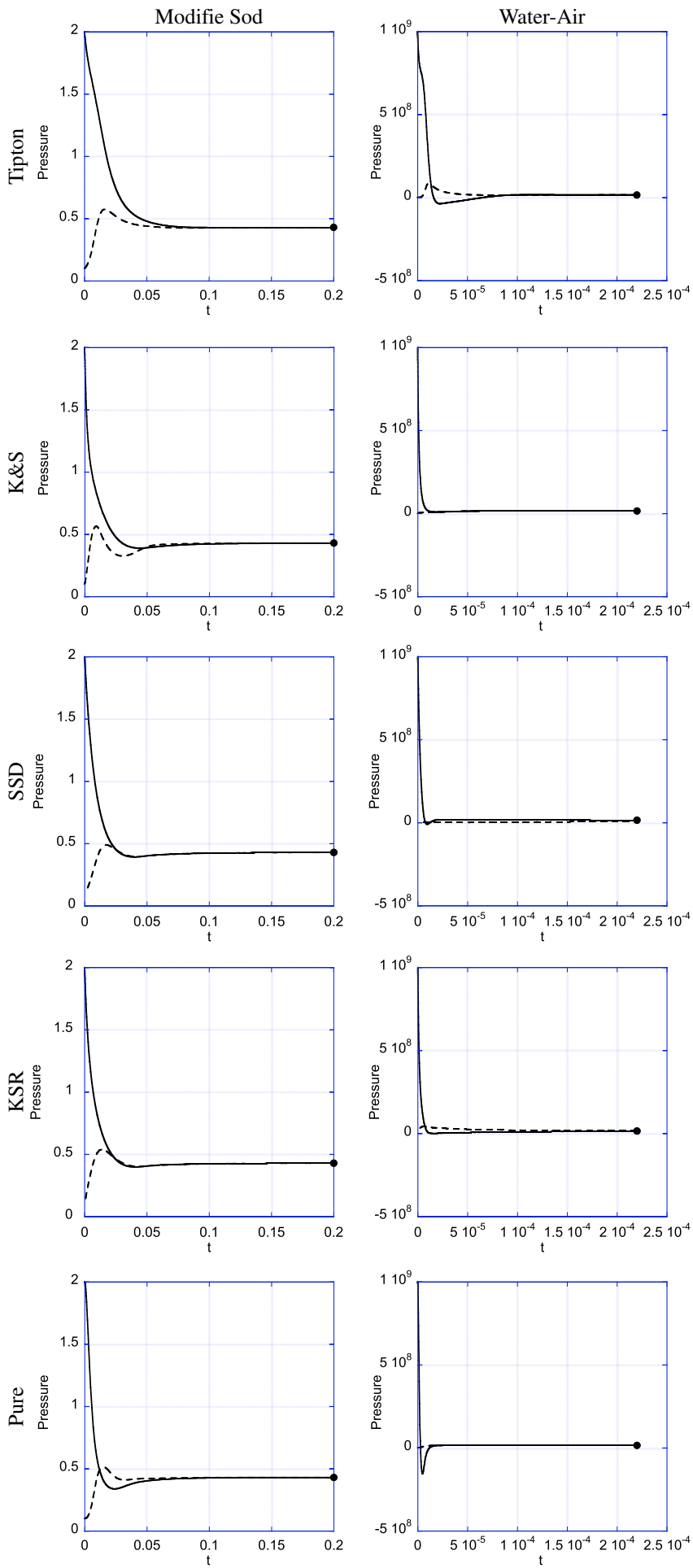

Fig. 1. Time-history plots for the pressure in the mixed cell for modified Sod shock tube problem (left column) and the water-air shock tube problem (right column) with Tipton's method, K\&S method, SSD method, the new KSR method and the pure-cell calculation. The top four rows are for two materials in the mixed cell; the bottom row is for the cells immediately adjacent to the material interface. The solid line indicates the left material (material 1), the dotted line represents the right material (material 2), and the bullets represent the exact solution at the final time. 
DE-AC52-06NA25396. The authors gratefully acknowledge the partial support of the US Department of Energy National Nuclear Security Administration Advanced Simulation and Computing (ASC) Program and the partial support of the US Department of Energy Office of Science Advanced Scientific Computing Research (ASCR) Program in Applied Mathematics Research. The authors thank A. Barlow, Yu. Bondarenko, D. Burton, B. Després, P.-H. Maire, L. Margolin, and Yu. Yanilkin for numerous stimulating discussions on these topics.

\section{References}

1. Barlow A., Proceedings of European Congress on Computational Methods in Applied Sciences and Engineering, ECCOMAS Computational Fluid Dynamics Conference, Swansea, Wales, U.K., 4-7 September 2001, 235-294

2. Delov V.I., Sadchikov V.V., VANT (Mathematical Modeling of Physical Processes) 1, (2005) 57-70 (in Russian)

3. Després B, Lagoutière F., Prog. Comput. Fluid Dyn. 7, (2007) 295-310

4. Goncharov E.A., Yanilkin Yu., VANT (Mathematical Modeling of Physical Processes) 3, (2004) 16-30 (in Russian)

5. Hirt C.W., Amsden A.A., Cook J.L., J. Comput. Phys. 14, (1974) 227-253

6. Kamm J.R., Shashkov M.J., Comm. Comput. Phys. 7, (2010) 927-976

7. Kamm J.R., Shashkov M.J., Fung J., Harrison A.K., Canfield T.R., Int. J. Numer. Meth. Fluids, (2010) (in press)

8. Saurel R., Abgrall R., J. Comput. Phys. 150, (1999) 425-467

9. Shashkov M.J., Int. J. Numer. Meth. Fluids 56, (2007) 1497-1504 